\title{
Recent progress in the performances of ultrastable quartz resonators and oscillators
}

\author{
Patrice Salzenstein* \\ Centre National de la Recherche Scientifique (CNRS), Franche Comté Electronique Mécanique Thermique Optique Sciences \\ et Technologies (FEMTO-ST) Institute, Université de Bourgogne-Franche-Comté (UBFC), 15B avenue des Montboucons, \\ 25030 Besancon, France
}

Received 18 November 2016 / Accepted 18 November 2016

\begin{abstract}
Stressed compensated (SC) cut led recently to the best frequency stability ever obtained with a quartz oscillator, $2.5 \times 10^{-14}$ for the flicker frequency modulation (FFM) floor. This result is confirmed in this paper with a $3.2 \times 10^{-14} \pm 1.1 \times 10^{-14}$. The quartz resonator is integrated in a $5 \mathrm{MHz}$ enhanced aging box double oven controlled oscillator. After reminding a bit of history, this paper describes how the first significant development in terms of ultra-stable quartz state-of-the-art oscillators was performed in the last 20 years, how the resonators were chosen, and main information about the development of adequate electronics and how to mechanically and thermally stabilized such an ultra-stable oscillator. We also present how to characterize the expected performances, and hot topics in quartz based oscillators.
\end{abstract}

Key words: Quartz, Frequency stability, Phase noise, Cut, Flicker frequency modulation floor.

\section{Introduction}

The piezoelectric effect is one of the particularity of quartz. Thanks to this effect, quartz has many applications especially in frequency standards sources and sensors. A mechanical deformation of this material appears when applying a voltage. It is useful for sensors as it concretely allows the probing of an acoustic resonance by electrical means. The application of alternating current to the quartz crystal induces oscillations. Standing waves are generated thanks to an alternating current between the electrodes of a properly cut crystal. The quality factor ( $Q$ factor) is the ratio of frequency and bandwidth. It can be as high as $2 \times 10^{6}$ for high quality quartz. Such a narrow resonance leads to highly stable oscillators and a high accuracy in the determination of the resonance frequency. The frequency of oscillation of a quartz crystal partially depends on its thickness. During normal operation, all the other influencing variables remain constant. Thus a change in the thickness of the material is directly correlated with a change in frequency. When fabricating the electrodes, mass is deposited on the surface of the crystal. The thickness then increases. Consequently the frequency of oscillation decreases from the initial value. The early history of the Development of ultra-precise oscillators for ground and space applications is strongly connected to the progress realized in quartz oscillators

*e-mail: patrice.salzenstein@femto-st.fr improvements [1]. In this paper we mainly focus on latter development. First the main quartz cuts are presented, then after a brief description of the structure use for achieving ultra stable quartz oscillators, we discuss the noise of the oscillators and how it is determined experimentally. Finally we say some words about how the obtained ultra stable signal can be distributed.

\section{Main different cuts in quartz}

\subsection{A bit of history before to present the main cuts}

For the very earlier history, let's remind that the first recorded experiments that relate to oscillators was performed by Jules Lissajous [2]. The piezoelectric effect was discovered by Jacques and Pierre Curie [3, 4]. Further works concerning electric oscillators are cited in reference [5] and well described in reference [6]. The first quartz oscillator was invented in 1921 [7]. In this section, we remind main information about some of the most common cuts. Resonator is obtained by performing cuts in the source crystal. Resonator plate can be cut from the source crystal in several ways. It has to be known that orientation of the cut influences several key parameters like thermal characteristics, crystal's aging characteristics, frequency stability and also other parameters. 


\subsection{AT-cut}

This cut can be considered as the main common cut. It is the older one as it was first developed in 1934. In this case, the plate contains crystal's $x$ axis. It is inclined by $35^{\circ} 15^{\prime}$ from the $z$ optic axis. Frequency versus temperature curves have typically a sine shaped with an inflection point at around $25-35{ }^{\circ} \mathrm{C}$. This cut is generally used for oscillating frequencies in the range $500 \mathrm{kHz}-300 \mathrm{MHz}$. It is used in wider temperature range. It is also used in oven-controlled oscillators.

\subsection{BT-cut}

This cut is similar to AT cut but used a different angle of $49^{\circ}$ from the $z$-axis. This cut allows repeatable characteristics. But its temperature stability characteristics are not as good as the AT-cut. The frequency operation range of the BT-cut is usually between $500 \mathrm{kHz}$ and $200 \mathrm{MHz}$. However it can be used for higher frequencies more easily than AT-cut.

\subsection{LD-cut}

The Low isochronism Defect (LD-cut) is a doubly rotated quartz resonator with a low amplitude-frequency effect called low isochronism defect. It is set in a enhanced aging box called in French Boîtier à Vieillissement Amélioré (BVA) structure for later use in an ultrastable oscillator (USO). Various design parameters were presented, along with the properties of the resonator. The resonance frequencies of various modes, the temperature dependence, the motional parameters and the phase noise in relation to the power supplied to the resonator were mainly studied [8].

\subsection{SC-cut}

The stressed compensated cut is called SC-cut. It is a double-rotated cut $\left(35^{\circ} 15^{\prime}\right.$ and $\left.21^{\circ} 54^{\prime}\right)$ for oven-stabilized oscillators with low phase noise and good aging characteristics. It is less sensitive to mechanical stresses. It has faster higher $Q$, warm-up speed and better phase noise close to the carrier. It has also less sensitivity to vibrations and less sensitivity to spatial orientation against the vector of gravity. This properties are useful for space and spacecraft applications. The frequency stability is determined by the crystal's loaded quality factor $\left(Q_{\mathrm{L}}\right)$. It is inversely dependent on the frequency. This cut is chosen to minimize temperature and frequency dependence [1]. Operating temperature of about $80-100{ }^{\circ} \mathrm{C}$ is needed for oven controlled crystal oscillators (OCXO) design. It is a double-rotated cut for oven stabilized oscillators with low phase noise, good aging characteristics and low sensitivity to drive level dependency [9]. It is generally used for oscillators operating for range of $0.5-200 \mathrm{MHz}$. Fabrication processes of SC-cut quartz resonators have now enough maturity, and allow to get ultra low noise crystal resonators. We see further that the very recent development of a new design for integrating a stressed compensated (SC) cut lead to significant progress for quartz frequency short term stability. It has to be underlined that recent study of new bulk acoustic waves (BAW) quartz resonators were performed $[10,11]$.

\section{Progress with BVA resonators}

For manufacturing a quartz oscillator, it is possible to use several resonator configurations by directly attaching leads to the crystal. Since 40 years BVA resonator has been giving the best frequency stability for USO. In 1976, a new class of resonator called BVA as it was already mentioned previously in the article was introduced by Besson [12]. The idea was to use a single crystal to reduce the mounting stress due to vibrations. Electrodes are deposited not on the resonator itself but on inner sides of two condenser discs made of adjacent slices of the quartz from the same bar. It forms a three-layer sandwich with no stress between the electrodes and the vibrating element. The resulting configuration is resistant to shock and vibration. It is also resistant to acceleration and ionizing radiation and has improved aging characteristics. Although AT cut is usually used, SC cut variants exist as well. BVA resonators are often used in spacecraft thanks to their characteristics. A tool can be used to sort out the best resonators showing its capability to be integrated into the best oscillator design [13]. It helps to choose best resonators for integration in an ultrastable oscillator. It contributes to decrease the influence of the noise [14]. One of the main contribution of the new design is certainly due to the hardware improvements in both of the oscillator and the electronics of the distribution amplifier system (DAS). First of all an ultra-stable enhanced aging box BVA oscillator is integrated with a DAS. The quartz resonator is mechanically stabilized by non adherent electrodes. It presents a double copper oven structure that considerably helps for thermal isolation of the quartz resonator, especially because it is compacted in a Dewar flask. The first oven is mechanically hold by a rigid composite material. The internal oven control card is placed in the second copper oven. The external card is placed inside the Dewar flask.

\section{Noise of the resonator}

The noise of the resonators to be integrated into BVA oscillators is typically measured on a bench developed for $5 \mathrm{MHz}$ resonators [15-17], France. Noise description has been detailed [18]. The main principle of such bench is to reject the split signal delivered by a $5 \mathrm{MHz}$ source into two arms. It is available at FEMTO-ST in Besançon, France. One is in quadrature and pumps the Local Oscillator (LO) input of the mixer. The other is separated in two other arms, each one owning a quasi-similar resonator. Tuning capacitors and attenuators permit the adjustment of frequencies and loading quality factors. These two parallel arms are shifted by $180^{\circ}$ in order to adjust suppression of the carrier. These two signals are then recombined, and the lateral bandwidths are amplified to the sufficient Radio Frequency (RF) input of the mixer, pumped by the reference signal in quadrature. Then, a Fast Fourier Transform (FFT) analyzer coupled to a Personal Computer (PC) allows us to obtain the spectral density of phase noise spectrum. Frequency of the resonators is temperature sensitive. It is important to be close to an inversion point where temperature has a minimal influence on the frequency. Hence, resonators to be measured are placed into appropriate thermostats [19]. For instance one resonator can have typically 
a temperature of inversion in the range $75-85^{\circ} \mathrm{C}$. Thermostats are especially developed for testing resonators and choosing the best of them, and of course also when they are integrated into an ultra stable oscillator. Thermostats for tests are very similar to the final packaging of oscillators in order to show, as closely as possible, the eventual working conditions of future oscillators.

For Fourier frequencies $f<f_{\mathrm{L}}$, where $f_{\mathrm{L}}$ is the cut-off frequency, the resonator filters its own frequency fluctuations leading to a $1 / f^{3}$ slope on the spectral density of phase noise $S_{\Phi}(f)$. Frequency cut-off of the resonator which is a low pass filter is $f_{\mathrm{L}}=v_{0} / Q_{\mathrm{L}}$ where $v_{0}$ and $Q_{\mathrm{L}}$ are respectively the carrier frequency - which here is typically chosen to be equal to $5 \mathrm{MHz}$ to obtain the most stable oscillators - and the loaded quality factor. Generally it can be noticed that the best performances in terms of frequency stability are obtained with more bulky quartz: Quartz at $2.5 \mathrm{MHz}$ or $5 \mathrm{MHz}$ are more stable than those dedicated to higher frequency. Introducing the Allan variance $\sigma_{\mathrm{y}}[20,21]$, we can then write that $S_{y}(f)=\left(f_{\mathrm{L}} /\right.$ $\left.v_{0}\right)^{2} \cdot S_{\Phi \mathrm{m}}(f)$. From $\sigma_{y}(\tau)=\sqrt{ }\left(2 \operatorname{Ln} 2 h_{-1}\right)$, with $h_{-1}=f_{\mathrm{L}}^{2} / v_{0}^{2}$. $S_{\Phi}(1 \mathrm{~Hz})$, we deduce that the frequency stability is at $1 \mathrm{~s}$ from the carrier. For a resonator chosen to present an example of measurement in this section, we have typically $Q_{\mathrm{L}}=$ $2.8 \times 10^{6}$. It has been measured with other resonators of the same batch. Indeed similar resonators generally present similar performances because they have been manufactured in the same batch. The curve is then obtained with the measurements of a resonator along with another from the same batch. If for instance, input power of each resonator is $60 \mu \mathrm{W}$, then spectral density of phase noise $S_{\text {? }}$ can be as lower as $S_{\Phi}(1 \mathrm{~Hz})=$ $-133 \mathrm{~dB} \mathrm{rad} / \mathrm{Hz}$. With a cut-off frequency $1.61 \mathrm{~Hz}$ on the chosen resonator in this part, the loaded quality factor is then $Q_{\mathrm{L}}=1.55 \times 10^{6}$. We can deduce that the frequency stability of the measured resonator pair is $8.5 \times 10^{-14} \pm 1.5 \times 10^{-14}$ at $1 \mathrm{~s}$ for the $5 \mathrm{MHz}$ carrier. Noise floor of the bench is $9.5 \times 10^{-15}$. If two resonators are exactly the same, frequency stability of each one could be obtained by dividing the obtained frequency stability of the pair by the square root of two. This approximation is generally valid if the resonators present sensibly the same performances. However if one of them presents worse stability, it will limit the obtained value of the frequency stability. So, the measured value is an upper value. Considering that the resonators have the same contribution, frequency stability of the resonator used to illustrate this part is $6.0 \times 10^{-14} \pm 1.5 \times 10^{-14}$ [13]. It is interesting to underline that this method gives an indication for sorting out the best resonators, despite if it is not that easy to perform such measure for each of the resonators when working in production for industry, but more generally in a context of research or control in a laboratory. But it is generally just enough to replace resonators in a blind oscillator, i.e. an oscillator where we just have to replace resonator by another one just by adjusting motional parameters and impedance adaptation of the crystal with its electronics. Finally frequency stability of the oscillator is really determined after packaging the resonator with electronics. It has to be underlined that the progress in terms of frequency stability of quartz oscillators are related to several multidisciplinary domains for optimization such has mechanical, thermal and electronic optimization.

\section{Stability Measurement of Oscillators}

\subsection{Determination of Frequency Stability for the Best Quartz Oscillators}

The main principle of the bench is based on Dual Mixer Time Difference Multiplication (DMTDM) [22] with a beat frequency of $5 \mathrm{~Hz}$. Such measures can be performed at the Institute of Photonics and Electronics (IPE) of the Czech Academy of Science in Prague. Each measure gives 10,000 samples. They are separated by a basic $200 \mathrm{~ms}$ integration time. When tested with the rejection of one BVA Oscillator, it is possible to deduce a flicker phase of $7 \times 10^{-15}$ at $1 \mathrm{~s}$. It can be negligible in the region where flicker floor is performed measuring between 1 and $100 \mathrm{~s}$. The resonator used to illustrate part 3 of this chapter is integrated into an oscillator. It is measured with other BVA oscillators. The best of them present flicker floor as low as $3.2 \times 10^{-14}$ [23] deduced by triangulation on several measures performed on different pairs of resonators [24]. The curve given in reference [24] shows a flicker floor at $7.5 \times 10^{-14}$ for integration times between $10 \mathrm{~s}$ and $100 \mathrm{~s}$. We can underline that even if those oscillators were carried under batteries during transport, each short disconnection can have an effect on the value of the stability. That's why performances of ultra stable quartz oscillators are typically guaranteed after enough time the quartz oscillators are being placed under supply voltage. It depends, but it is better to wait $24 \mathrm{~h}$ or even 1 week before to obtain the best performances in terms of noise floor and frequency stability. At $1 \mathrm{~s}$, frequency stability of the chosen pair can be estimated to be $9.5 \times 10^{-14}$. The two oscillators present similar performances. This is why, in a first approximation, their contribution to the measured noise can be considered as equal. An estimation of the value of the stability of one oscillator can then be $6.7 \times 10^{-14}$ at $1 \mathrm{~s}$, with a flicker frequency modulation (FFM) floor at $5.5 \times 10^{-14}$ between $20 \mathrm{~s}$ and $100 \mathrm{~s}$. We can attribute these values with an uncertainty equal to $\pm 3 \times 10^{-15}$. We note that the accuracy of the Three-Cornered Hat method [25-27] is discussed when not simultaneously applied to the determination of the contribution on short term frequency stability performed on ultra-stable quartz oscillator. It led to a validation of the results [28].

\subsection{State-of-the-art}

There have been no significant changes in the noise floor of commercial oscillators in the years 1995-2010: the very best commercial quartz oscillators operate with a short-term frequency stability of $8 \times 10^{-14}$. But the best frequency stability ever measured on a quartz crystal oscillator was then obtained in 2010. This new BVA oscillator has an estimated flicker frequency modulation (FFM) floor of $2.5 \times 10^{-14}$ at $5 \mathrm{MHz}$ [29]. It was underlined that it leads to a significant step [30]. It was obtained using a double rotated SC-cut quartz with low phase noise and good aging characteristics and low sensitivity to drive level dependency [9] placed in appropriate thermostat [19] in the first prototype of an Oven-controlled crystal oscillator (OCXO) realized in Switzerland by Oscilloquartz company. Such frequency stability is equivalent 


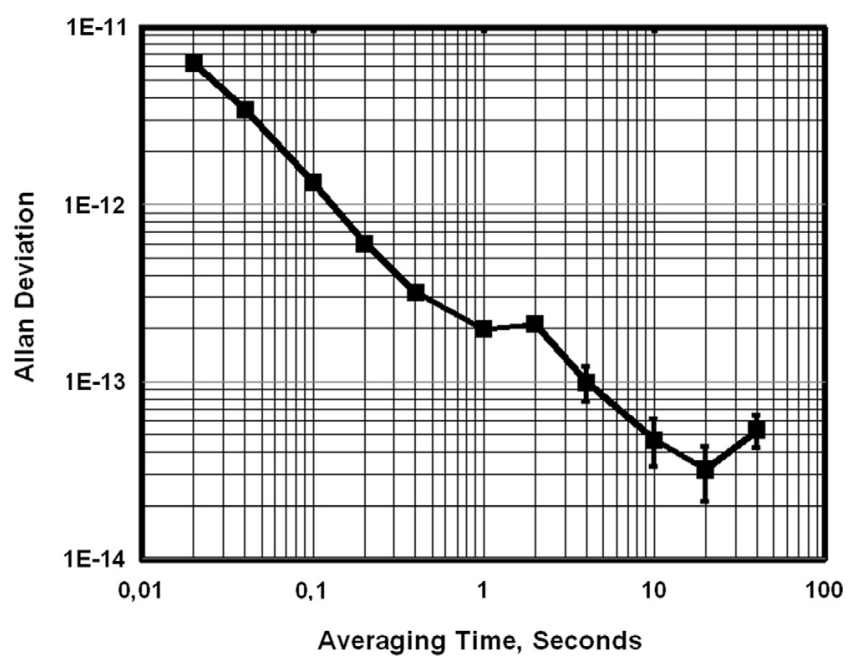

Figure 1. Frequency stability of two $5 \mathrm{MHz}$ BVA quartz measured with time interval analyzer. The Allan deviation $\sigma_{y}(\tau)$ is given versus averaging integration time $\tau$. This quartz resonator presents a FFM floor of $3.2 \times 10^{-14} \pm 1.1 \times 10^{-14}$ at $5 \mathrm{MHz}$ determined for a $\tau=20 \mathrm{~s}$

to a variation of only one second during $1.3 \times 10^{6}$ years, but measured in terms of frequency stability for a few seconds integration time, as the main interest of such oscillators is to deliver an ultra stable signal on short term. The recent results obtained show that it was too early to "bury" research into quartz and that it certainly has the potential to reach the level of $1 \times 10^{-14}$. The frequency stability curve of a recent BVA USO measured in FEMTO-ST, Besançon, France is given in Figure 1. The measured FFM floor is $3.2 \times 10^{-14} \pm$ $1.1 \times 10^{-14}$ at $5 \mathrm{MHz}$. It is obtained for a $20 \mathrm{~s}$ averaging integration time. This measurement result and its associated uncertainty are coherent with the best FFM floor previously measured [29]. We can notice that the oscillator aging hides the FFM floor for upper integration time.

\section{Distribution of the signal}

In this section, we detailed how such an ultra stable signal can be distributed in a laboratory. To illustrate the level of performances, we choose concretely a distribution amplifier system realized in Besançon. The amplifier stage is significantly improved thanks to the multiplication by two, that is the doubling. It allows an optimal performance in power combined with an excellent noise factor. It is achieve by using the rectifier effect of a diode bridge. Spurious peaks are filtered by low pass filters, especially at 20 and $30 \mathrm{MHz}$ for the $10 \mathrm{MHz}$ output as the level of output signals at 5, 10, 15, 20, 25, $30 \mathrm{MHz}$ were respectively $-45,8,-41,-8,-47,-18 \mathrm{dBm}$ before any filters. After the filters, power at 20 and $30 \mathrm{MHz}$ are lead to $-76 \mathrm{dBc}$ and $-91 \mathrm{dBc}$.

The evaluation of the performance is not an easy point to understand, and that's why it has to be explained a little bit more. It is a critical point. The fact to have a minimum of two different outputs for each frequency helped for characterization and for comparison of the influence of the connectors,

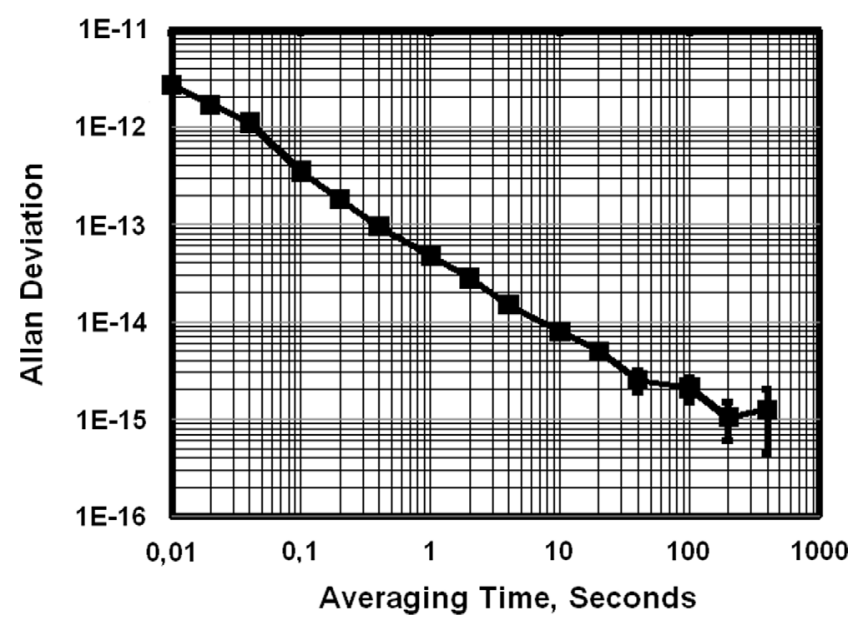

Figure 2. Distribution amplifier measured Allan variance at $10 \mathrm{MHz}$ on a time interval analyzer.

but this is not so critic compared to the ability to determine the noise floor of the instrument and to measure the performance of the realized DAS: we present in Figure 2 the results of the noise floor of the measurement setup with a "Timing Solutions" TSC 5110A.

Allan variance obtained by this method is $4.8 \times 10^{-14}$ and $2.3 \times 10^{-14}$ at $5 \mathrm{MHz}$ and $10 \mathrm{MHz}$ for a $1 \mathrm{~s}$ integration time. The results obtained in terms of Allan variance are in that case identical to the noise floor provided by the manufacturer of the dynamo-meter used, that is to say $5 \times 10^{-14}$ and $2.5 \times 10^{-14}$ respectively at $5 \mathrm{MHz}$ and $10 \mathrm{MHz}$ for a $1 \mathrm{~s}$ integration time. In our case, the measurement of the Allan variance is actually calculated automatically from the compilation of measures from the frequency counting. However, we find that the measure is apparently limited by the performance of such a bench. With such a method it is not possible to accurately assess the performance of the distribution amplifier in terms of short-term frequency stability. Indeed we achieve the evaluation of the noise floor of our system by performing phase noise measurements. It is given in Figure 3.

Considering that both channels have a similar contribution, the values at $1 \mathrm{~Hz}$ from the carrier are as follows.

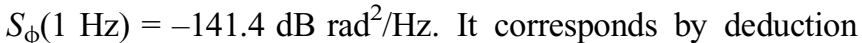
to $1.4 \times 10^{-14}$ for $1 \mathrm{~s}$ integration time at $5 \mathrm{MHz}$ and similarly $7.3 \times 10^{-14}$ for $1 \mathrm{~s}$ at $10 \mathrm{MHz}$. The performances have then been fully characterized with a Dual Mixer Time Difference Multiplication (DMTDM) based system described in the article and in some of the references [13, 22]. In our case, we were able to measure the real performance of the distributed signals with a flicker frequency modulation (FFM) equal to $4.5 \times 10^{-14}$ at $12 \mathrm{~s}$. We are certain that this is the real value, as this reference quartz was previously compared to the best quartz oscillator that was ever manufactured and presented in reference published in 2010 [29]. Additionally, fact that the noise floor better than the best performance ever measured on a quartz oscillator enable the use of such a DAS for future characterizations. This recent development are presented in references [31]. Such quartz are used in laboratories for metrology applications. When there 


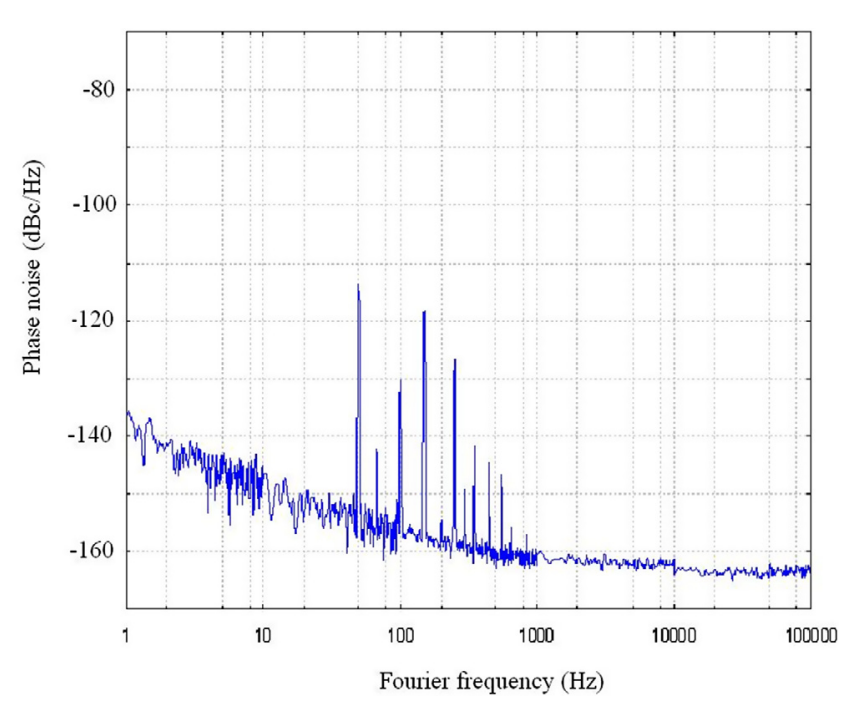

Figure 3. Noise floor of our system measured for two DAS in $\mathrm{dBc} / \mathrm{Hz}$.

characteristics fit with space requirements, they can be used for space applications.

\section{Hot topics}

Results published in the last months shows an overview of the hot topics in quartz research. Some researchers present new reliable quartz oscillator technology [32]. Progress comes also from lower temperature of operation with Cryogenic Quartz Oscillators [33]. Fundamental resonance frequency dependence of the proximity effect of quartz crystal resonators was demonstrated [34]. $1 / \mathrm{f}$ noise in quartz resonators is still a hot topic [35]. For experimental use, uncertainty calculation is better known with a modern way of estimating thanks to Guide to the Expression of Uncertainty in Measurement (GUM) edited by the Bureau International des Poids et Mesures (BIPM), intergovernmental organization through which Member States act together on matters related to measurement science and measurement standards [36]. Typical uncertainty calculation on phase noise in the RF domain are given in references $[37,38]$.

\section{Conclusion}

In this paper were presented the recent progress in Quartz oscillators fabrication and instrumentation to enable measure of the best frequency stability performances on resonators and oscillators. It especially led to the first significant development in the last 15-25 years in terms of ultra-stable quartz state-of-the-art oscillators. If the best oscillator was realized in Switzerland, instrumentation was improved in France and in Czech Republic. It especially includes the choice of the resonators, the development of adequate electronics and how to mechanically and thermally stabilized such an ultra-stable oscillator, but also state-of-the-art frequency stability on oscillators. The last section of this paper also presented the latest development for distributing such ultra stable signals.
The best flicker floor is as low as $2.5 \times 10^{-14}$ measured by Three-Cornered Hat method in Prague and confirmed at $3.2 \times 10^{-14} \pm 1.1 \times 10^{-14}$ by direct time interval analyzer in Besançon. It shows that it is certainly too early to "bury" research into quartz as it certainly has the potential to reach the level of $1 \times 10^{-14}$ in future. This objective has not been yet achieved, but research is still active in the field of quartz oscillators.

Acknowledgements. Author thanks Dr. Alexander Kuna, head of the Laboratory of the National Time and Frequency Standard at the Institute of Photonics and Electronics and Academy of Science in Prague (Czech Republic) and Dr. Jacques Chauvin, formerly at Oscilloquartz Neuchatel (Switzerland). This work was supported by the Laboratoire national de métrologie et d'essai (LNE).

\section{References}

1. Norton JR, Cloeren JM, Sulzer PG. 1996. Brief history of the development of ultra-precise oscillators for ground and space applications, in Proceedings of the IEEE Int. Freq. Contr. Symp., p. 47-57.

2. Lissajous J. 1857. Mémoire sur l'étude optique des mouvements vibratoires. Comptes Rendus, 44, 727.

3. Curie J, Curie P. 1880. Développement par pression, de l'électricité polaire dans les cristaux hemiedres à faces inclinées. Comptes Rendus, 91, 294.

4. Curie J, Curie P. 1882. Déformations électrique du quartz. Comptes Rendus, 95, 914-917.

5. Dadourian HM. 1919. On the characteristics of electrically operated tuning forks. Physical Review, 13, 337-359.

6. Marrison WA. 1948. The evolution of the quartz crystal clock. Bell System Technical Journal, 27(3), 510-588.

7. Cady WG. 1922. The piezoelectric resonator. Proceedings of the Institute of Radio Engineers, 10, 83-114.

8. Gufflet N, Sthal F, Boy JJ, Bourquin R, Mourey M. 2001. Doubly rotated quartz resonators with a low amplitudefrequency effect: the LD-cut. IEEE Transactions on Ultrasonics, Ferroelectrics, and Frequency Control, 48(6), 1681-1685.

9. Brendel R, Addouche M, Salzenstein P, Rubiola E, Shmaliy YS. 2004. Drive level dependence in quartz crystal resonators at low drive levels: a review, in Proc. of the 18th European Frequency and Time Forum, Guilford, UK, IEE Conf. Pub., CP499. p. 11-18.

10. Clairet A. 2014. Modelisation et analyse numérique de resonateurs a quartz a ondes de volume, $\mathrm{PhD}$ Thesis, University of Franche Comte.

11. Clairet A, Laroche T, Couteleau L, Boy JJ. 2013. Experimental and theoritical results on SC-cut quartz resonators collectively realized on 4" wafers, in 2013 Joint European Frequency and Time Forum \& International Frequency Control Symposium (EFTF/IFC), Prague, Czech Republic. p. 662-665.

12. Besson R. 1977. A new electrodeless resonator design, in Proc. 31st Ann. Symp. on Frequency Control, June 1977. p. 147-152.

13. Salzenstein P, Kuna A, Sojdr L, Sthal F, Cholley N, Lefebvre F. 2010. Frequency stability measurements of ultra-stable BVA resonators and oscillators. Electronics Letters, 46(10), 686-688. 
14. Walls FL. 1995. The quest to understand and reduce $1 / \mathrm{f}$ noise in amplifiers and BAW quartz oscillators, in Proc. of the 9th European Freq. and Time Forum, Besançon, France. p. 227-240.

15. Sthal F, Mourey M, Marionnet F, Walls WF. 2000. Phase noise measurements of $10 \mathrm{MHz}$ BVA quartz crystal resonator. IEEE Trans. on Ultrason. Ferroelec. Freq. Contr., 47(2), 369-373.

16. Sthal F, Vacheret X, Salzenstein P, Galliou S, Rubiola E, Cibiel G. 2007. Advanced bridge instrument for the measurement of the phase noise and of the short-term frequency stability of ultrastable quartz resonator, in Proceedings of the IEEE Freq. Contr. Symp. \& European Freq. and Time Forum, Geneva, Switzerland. p. 254-260.

17. Walls FL. 1997. Suppressed carrier based PM and AM noise measurement techniques, in Proceedings of the IEEE Freq. Contr. Symp, p. 485-492.

18. Sthal F, Imbaud J, Devel $M$, Salzenstein $P$, Bourquin R, Cibiel G. 2011. Some considerations on acoustic resonator phase noise modeling and recent short-term stability experimental results, in Frequency Control and the European Frequency and Time Forum (FCS), 2011 Joint Conference of the IEEE International, San Francisco, California, USA, 2-5 May.

19. Sthal F, Galliou S, Abbe P, Vacheret G, Cibiel G. 2007. Ultra stable crystal ovens and simple characterization. Electronics Letters, 43(16), 900-901.

20. Gray JE, Allan DW. 1974. A Method for Estimating the Frequency Stability of an Individual Oscillator, in Proc. of the 28th Ann. Symp. on Freq. Contr, May 1974. p. 243-246.

21. Allan DW. 1987. Time and frequency (time domain) characterization, estimation, and prediction of precision clocks and oscillators. IEEE Trans. on UFFC, 34(6), 647-654.

22. Brida G. 2002. High resolution frequency stability measurement system. Review of Scientific Instruments, 73(5), 2171-2174.

23. Kuna A, Cermak J, Sojdr L, Salzenstein P, Lefebvre F. 2010. Lowest flicker-frequency floor measured on BVA oscillators. IEEE Ultrasonics, Ferroelectrics, and Frequency Control Society, 57(3), 548-551.

24. Cermak J, Kuna A, Sojdr L, Salzenstein P. 2007. Short-Term Frequency Stability Measurement of BVA Oscillators, in Proc. of the IEEE Freq. Contr. Symp. \& European Freq. and Time Forum, Geneva, Switzerland. p. 1255-1260.

25. Ekstrom CR, Koppang PA. 2006. Error bars for three-cornered hats. IEEE Transactions on Ultrasonics, Ferroelectrics and Frequency Control, 53(5), 876-879.

26. Vernotte F, Addouche M, Delporte M, Brunet M. 2004. The three cornered hat method: an attempt to identify some clock correlations, in IEEE Freq. Contr. Symp., 23-27 Aug., 2004. p. $482-488$.

27. Tavella P, Premoli A. 1993. Estimation of instabilities of $\mathrm{N}$ clocks by measuring differences of their readings. Metrologia, 30(5), 479-486.

28. Salzenstein P, Kuna A, Lefebvre F. 2013. Evaluation of the accuracy of the method for measuring state-of-the-art ultra-high stability quartz crystal oscillators, in 2013 Joint European Frequency and Time Forum \& International Frequency Control Symposium (EFTF/IFC), Prague, Czech Republic. p. 157-159.

29. Salzenstein P, Kuna A, Sojdr L, Chauvin J. 2010. Significant step in ultra high stability quartz crystal oscillators. Electronics Letters, 46(21), 1433-1434.

30. Dyball H. 2010. Dropping through the floor. Electronics Letters, 46(21), 1411.

31. Salzenstein P, Cholley N, Kuna A, Abbé P, Lardet-Vieudrin F, Sojdr L, Chauvin J. 2012. Distributed amplified ultrastable signal quartz oscillator based. Measurement, 45(7), 1937-1939.

32. Lo A, Haslinger P, Mizrachi E, Anderegg L, Müller H. 2016. Acoustic tests of Lorentz symmetry using quartz oscillators. Physical Review X, 6, 011018.

33. Goryachev M, Ivanov EN, Tobar ME, Galliou S. 2016. Towards Cryogenic Quartz Oscillators: Coupling of a Bulk Acoustic Wave quartz resonator to a SQUID, in Frequency Control Symposium (IFCS), 2016 IEEE International Date of Conference, 9-12 May.

34. Yao Y. 2015. Fundamental resonance frequency dependence of the proximity effect of quartz crystal resonators. Japanese Journal of Applied Physics, 54(11), 6701.

35. Sthal F, Devel M, Imbaud J, Bourquin R, Ghosh S, Cibiel G. 2016. Study on the origin of $1 / \mathrm{f}$ noise in quartz resonators. Journal of Statistical Mechanics: Theory and Experiment, 2016, 054025 .

36. GUM: Guide to the Expression of Uncertainty in Measurement, fundamental reference document, JCGM100:2008 (GUM 1995 minor corrections), http://www.bipm.org/en/publications/guides/ gum.html.

37. Salzenstein P, Pavlyuchenko E, Hmima A, Cholley N, Zarubin M, Galliou S, Chembo YK, Larger L. 2012. Estimation of the uncertainty for a phase noise optoelectronic metrology system. Physica Scripta, T149, 014025.

38. Salzenstein P, Wu TY. 2016. Uncertainty analysis for a phasedetector based phase noise measurement system. Measurement, $85,118-123$.

Cite this article as: Salzenstein P: Recent progress in the performances of ultrastable quartz resonators and oscillators. Int. J. Simul. Multisci. Des. Optim., 2016, 7, A8. 